\title{
Progress in the construction of the CDF silicon vertex detector
}

Presented by J.E. Skarha

\author{
B. Barnett, C. Boswell, J. Matthews ' , J.E. Skarha, F.D. Snider, A. Spies, J. Tseng \\ and S. Vejcik
}

The Johns Hopkins Unicersity, Baltimore MD 21218, USA

H. Carter, B. Flaugher, B. Gonzales, M. Hrycyk, C. Nelson, S. Segler, T. Shaw, P. Tipton, S. Tkaczyk and K. Turner

Fermi National Accelerator Laboratory, Bataicia IL 60510, USA

W.C. Carithers, R. Ely, M. Gold ', C. Haber, S. Holland, S. Kleinfelder, T. Merrick, O. Schneider, W. Wester and M. Wong

Lawrence Berkeley Laboratory, Berkeley CA 94720, USA

\section{Amidei and P.F. Derwent}

Unicersity of Michigan, Ann Arbor MI 48109, USA

\author{
N. Bacchetta, D. Bisello, G. Busetto, A. Castro, M. Loreti and L. Pescara \\ Unicersity of Padoia, I-35100 Padoia, Italy
}

\author{
F. Bedeschi, V. Bolognesi, S. Dell'Agnello, S. Galeotti, M. Mariotti, A. Menzione, G. Punzi, \\ F. Raffaelli, L. Ristori, F. Tartarelli, N. Turini, H. Wenzel and F. Zetti \\ Istituto Nazionale di Fisica Nucleare, Unicersity and Scuola Normale Superiore of Pisa, 1-56100 Pisa, Italy
}

\author{
M. Bailey, A.F. Garfinkel and N.M. Shaw \\ Purdue Unicersity, West Lafayette IN 47907, USA
}

\begin{abstract}
The mechanical design and construction progress of the CDF silicon vertex detector is described. Results on the location accuracy of the silicon strip detectors are presented and indicate an initial placement uncertainty of less than $10 \mu \mathrm{m}$. The water and gas cooling system, which is constructed of low mass materials and is used to remove heat from the readout electronics, is briefly described. Measurements of the performance of the silicon strip detectors are also given and show $>\mathbf{9 8 . 5 \%}$ functioning strips for the assembled SVX detector.
\end{abstract}

\section{Introduction}

A silicon strip vertex detector ("the CDF SVX") is being constructed for use with the CDF detector at the Fermilab Tevatron $\bar{p} \mathbf{p}$ collider. This detector will measure the impact parameter of tracks from the decay of

1 Now at the University of New Mexico. Albuquerque, New Mexico 87131, USA. long-lived particles $(c \tau>300 \mu \mathrm{m})$. Of particular interest is the measurement of the properties of $B$ mesons produced either directly in the proton-antiproton collision or from the subsequent decay of a top quark. In this paper, we review the mechanical design and construction of the SVX and present results from the initial mechanical alignment. A description of the cooling system used to remove heat from the detector volume is presented and the performance of the silicon strip detectors is also described. Recent test beam 
results [1] and radiation damage tests to the readout chip [2] are described in other contributions to this conference (5th Pisa Meeting on Advanced Detectors).

\section{SVX design considerations}

The CDF SVX detector was designed under the rigid requirements imposed by operation at a hadron collider. These constraints immediately forced certain design choices. At the Tevatron collider, the p p event vertex in the CDF collision hall is Gaussian distributed along the beamline with $\sigma=35 \mathrm{~cm}$. Thus a fairly long detector is required in order to have good event acceptance. This is compared to experiments at an $\mathrm{e}^{+} \mathrm{e}^{-}$ collider where the particle bunch lengths are typically much shorter. The SVX detector is $51 \mathrm{~cm}$ in length and will contain $\sim 60 \%$ of the $\bar{p} p$ collision vertices.

The amount of material used to construct the SVX must be kept to an absolute minimum for successful reconstruction of the high multiplicity $\bar{p} p$ events. This is because creation of secondary particles and conversion pairs in any additional material will be a source of background for all CDF detectors and multiple scattering is the limiting factor in measuring the impact parameter of low momentum tracks. Also, the materials involved need to be mechanically stable and robust in the relatively high radiation environment of a hadron collider.

The scale of the mechanical tolerances required in the construction of the detector must be comparable to the intrinsic position resolution of the strip detectors. For detectors with a $60 \mu \mathrm{m}$ strip pitch, the intrinsic position resolution is $17 \mu \mathrm{m}$ and can go as low as 10 $\mu \mathrm{m}$ with charge sharing information on neighboring strips. In order to achieve position information with similar resolution in the completed SVX detector, the construction errors must then be on order $10 \mu \mathrm{m}$ or less. This is a challenging task and requires great care in the choice of materials and assembly techniques. Simulations of the detector in the high multiplicity hadron collider environment led to the design decision to install four lavers of silicon, rather than the two or three layers used in previous experiments. The four layers of silicon allow an additional level of redundancy which greatly reduces hit misassociation in the track

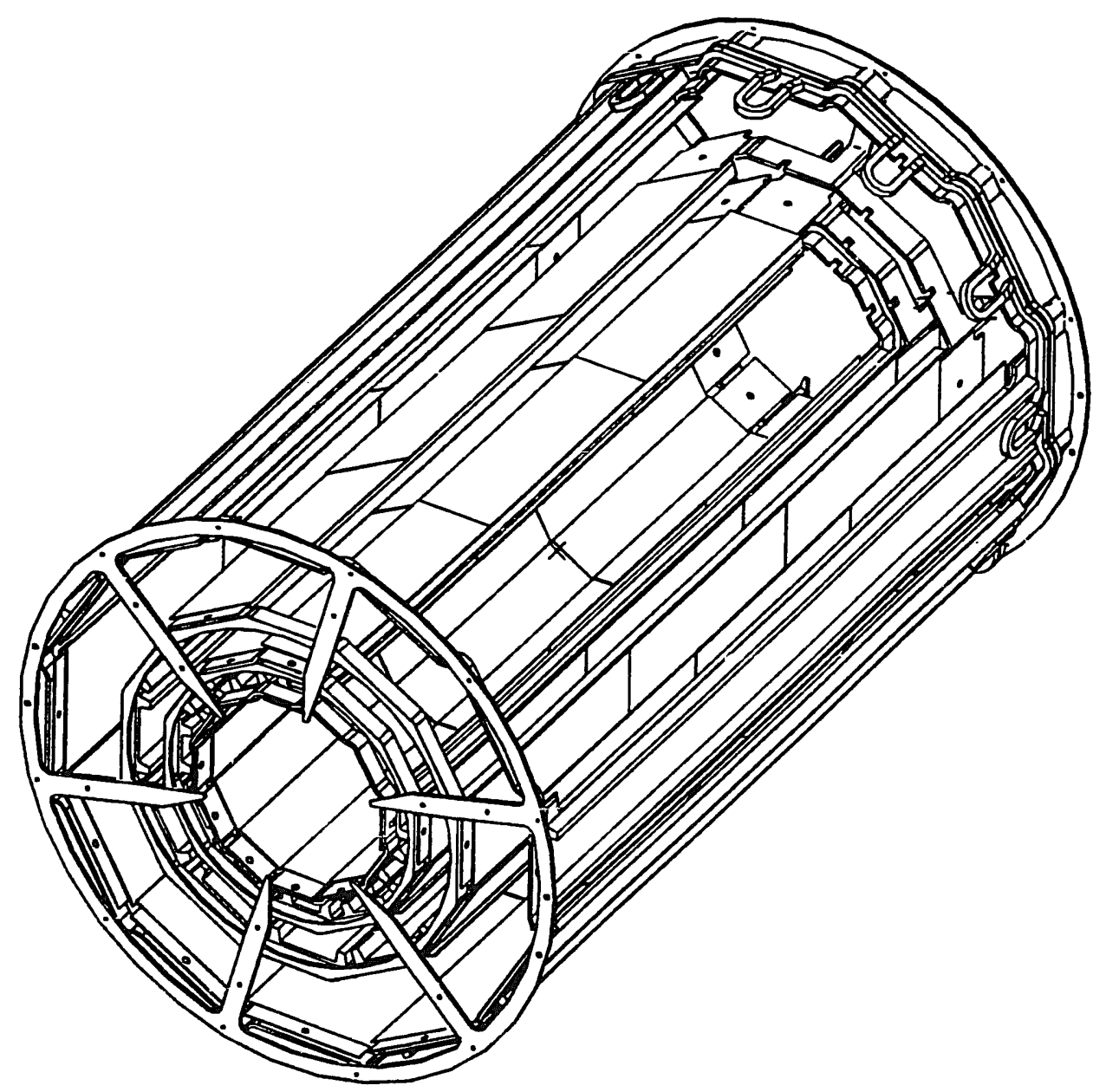

Fig. 1. Schematic view of one-half of the SVX detector, showing the internal geometry. 
segment finding and improves significantly the matching of track segments in the silicon detector with tracks found in the CDF outer tracking chambers.

Finally, the front-end electronics must be able to integrate and store the signal from a single strip in less than the $3.5 \mu$ s crossing time between $\bar{p}$ p collisions. It must also contain a sparse threshold function in order to limit the amount of data recorded and to avoid incurring significant readout deadtime. It is expected that the readout time of the completed SVX detector will be only $1.8 \mathrm{~ms}$.

\section{SVX mechanical design and alignment}

The CDF silicon vertex detector has been described previously [3]. Here, we present updates to the design and additional details on the construction of the detector. Fig. 1 shows one-half of the SVX detector. The detector consists of two barrel modules placed end-toend which are centered on the nominal interaction collision point and whose axes are coincident with the beam axis. Contained inside these cylindrical modules are four radial layers of silicon strip detectors arranged in a 12-sided geometry. The detectors are $300 \mu \mathrm{m}$ thick and have strip pitch of wi) $\mu \mathrm{m}$ for the three layers nearest to the beam and $55 \mu \mathrm{m}$ for the fourth layer. The detector width increases with radius to provide a wedge geometry which points back to the beamline. The detectors are electrically bonded to each other along the beam direction in groups of three. These bonds are made with $25 \mu \mathrm{m}$ diameter aluminum wire, which contains $1 \%$ silicon in order to reduce electrochemical diffusion of the aluminum into the silicon detector, to stiffen the wire and to retain heat during the ultrasonic welding of the wire to the pads on the detector surface. The three detectors are read out electronically at one end. Each detector is $8.5 \mathrm{~cm}$ long. An individual readout channel is therefore connected to a $25.5 \mathrm{~cm}$ long strip which has a capacitance of $\sim 30$ pF.

The three detectors are glued to a lightweight Rohacell [4] and carbon fiber support structure. This combination of detectors and support structure is known as a "ladder". Fig. 2 shows the layout of an SVX ladder. The Rohacell is a polymethacrylimide rigid foam of very low density with good mechanical properties. This foam is first milled to the desired dimensions and then carbon fiber strips are epoxied into it using a compression mold heated to $125^{\circ} \mathrm{C}$. These ladder substrates are typically made flat to within $75 \mu \mathrm{m}$. During the detector gluing operation, the detectors are placed on top of the ladder substrate and are held firmly in a specially designed gluing fixture utilizing a vacuum chuck. Since the strips of the three detectors on a ladder are connected electrically, it is necessary that the detectors are aligned to one another to better than their intrinsic position resolution. To accomplish this, the gluing fixture is mounted on a granite table and the detectors are aligned by focussing on the strips with a high-magnification TV camera mounted on the measuring arm of a coordinate measuring machine (CMM) [5] and moving them into position with micrometer adjustable stops. The reference

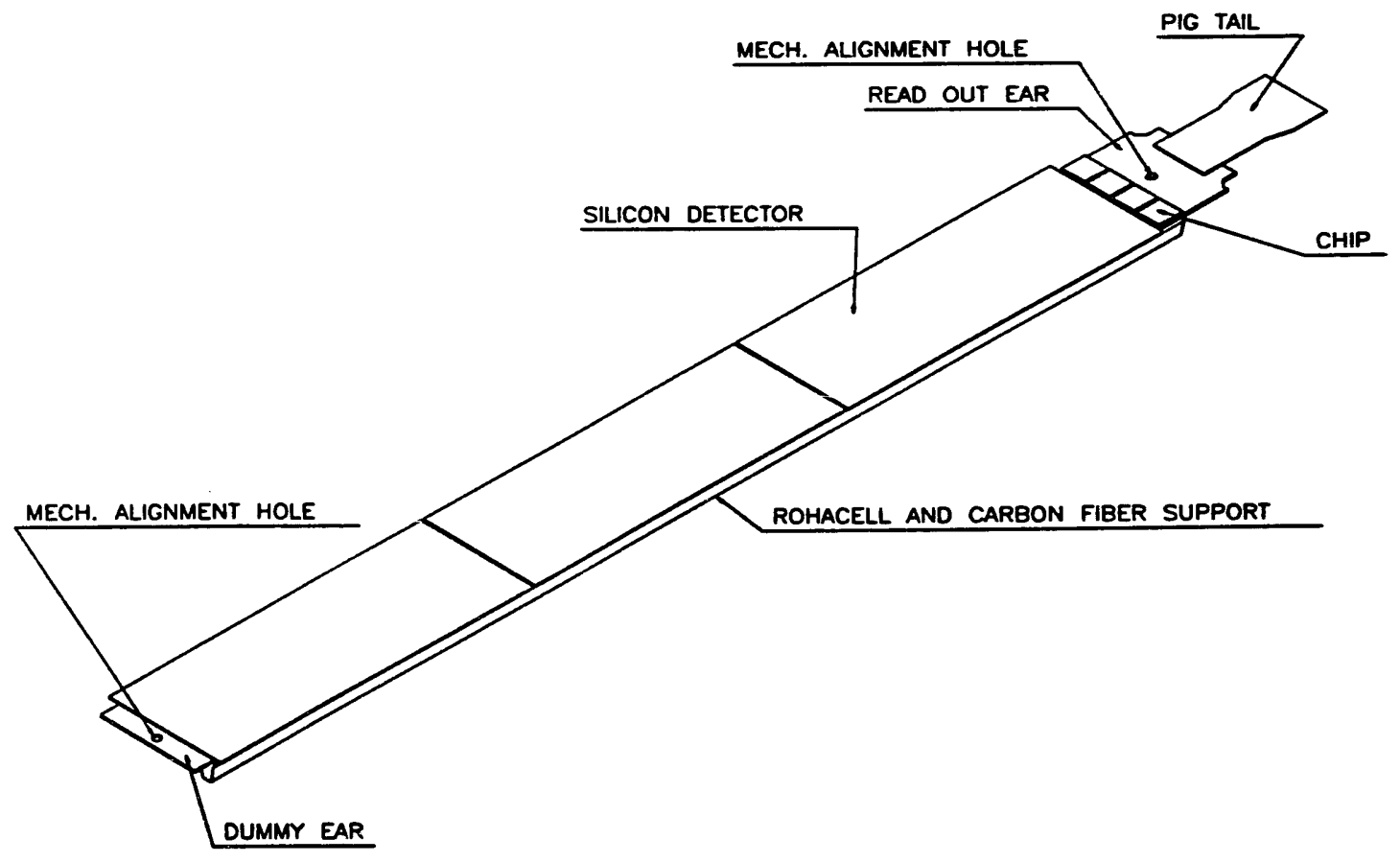

Fig. 2. Components of an SVX ladder module. 


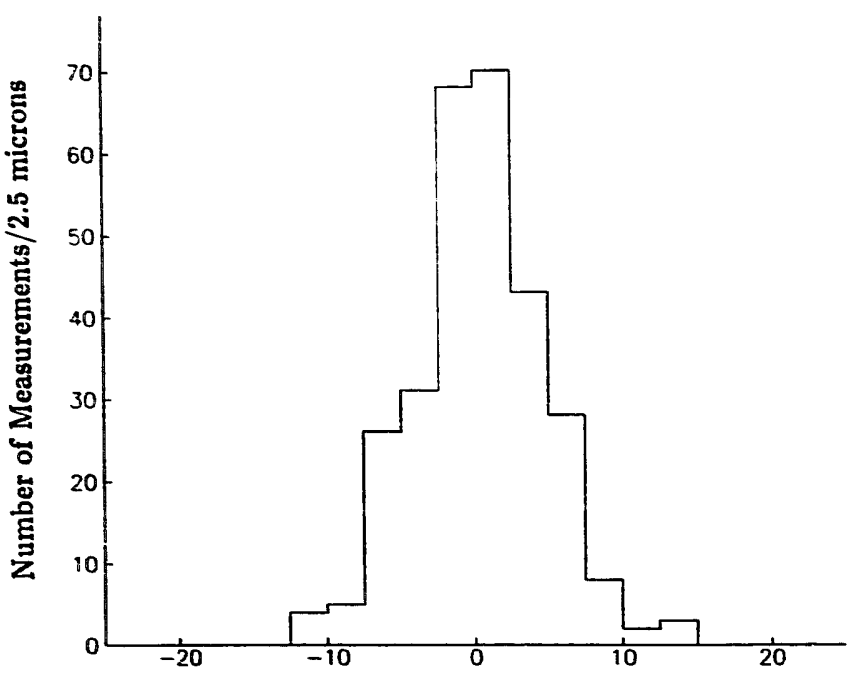

Barrel \#1 Ladder Detector Alignment (microns)

Fig. 3. Detector alignment for all ladders mounted in the first SVX barrel.

line for the detector alignment is a line, established by the CMM software, through the centers of the laserdrilled mounting holes in the circuit boards at both ends of the ladder. Each of the three detectors are adjusted so that their center strip is aligned to this reference line. The epoxy [6] used to glue the detectors to the ladder substrate exhibits very little shrinkage or outgassing during the low temperature $\left(60^{\circ} \mathrm{C}\right.$ for two hours) heat curing process. Fig. 3 shows that the detector alignment after gluing has an rms of $4.4 \mu \mathrm{m}$ and is consistent with the $5 \mu \mathrm{m}$ measurement repeatability calibration of the CMM.

Each SVX ladder was individually assembled in an overpressured Class 100000 clean room in which the room temperature and humidity were monitored and controlled. Standard clean room apparel, such as a rubber gloves, a face mask and a hair bouffant, were wore by the technician constructing the ladder. The total number of ladders in the SVX detector is 12 faces $\times 4$ layers $\times 2$ ends $=96$ ladders or 24 ladders of each of the four radial types. In its final mounted position in the barrel, each ladder is rotated by $3^{\circ}$ about its length in order to allow overlap between adjacent ladders and to minimize azimuthal boundary gaps.

One of the circuit boards (also known as "ears") mounted on the ladder contains the SVXD readout chips on a thick-fiim aluminum nitride substrate. The aluminum nitride material [7] has a thermal conductivity of $160 \mathrm{~W} / \mathrm{m}^{\circ} \mathrm{K}$ which is similar to that of metallic aluminum and was used to provide good conduction of the heat from the chips to the cooling system. The coefficient of thermal expansion of aluminum nitride is also well matched to that of silicon. Care has been taken to make sure that the readout ear is thermally isolated from the nearest silicon detector in order to prevent conduction of heat from the chips directly into the silicon. At the opposite end of the ladder, a second "dummy" ear is used for mounting the ladder into the barrel.

The SVXD chip itself was fabricated using $3 \mu \mathrm{m}$ feature size CMOS technology and has both a digital and analog section [8,9]. The analog section contains 128 channels of charge-integrating amplifiers in double or quadruple correlated sample and hold readout scheme.

The readout cable (known as a "pigtail") attached to the end of the readout ear board incorporates a "gold-dot technology" [10] to make electrical contact to a mating bus cable. This bus cable connects the four ladders in a wedge to a driver/filtering board (known as the "port card") located just above the fourth layer of silicon inside the barrel. Small bumps or dots of gold have been deposited on the traces at the end of the flexible Kapton pigtail cable. These bumps then mate with the bus cable which contains pads using a lightweight G-10 clamp fastened with a small bolt and nut. The interconnect pitch used has traces on staggered 40 mil centers, giving an effective 20 mil pitch. This connection scieme allows easy assembly of the cables and works well compared to the conventional pin and socket arrangement which is only available for traces on no smaller than a $\mathbf{5 0}$ mil pitch. Furthermore, this scheme allowed complete flezibility in the number of traces and in the cable layout.

The SVX ladders are installed between two support pieces known as "bulkheads" using a small screw, O-ring and tapped pin combination which fits through the mounting holes of the ears and attaches to slots machined into the ledges of the bulkheads. The slots in the bulkhead allow the ladder to expand thermally along its length while maintaining good azimuthal location. These bulkheads were made from beryllium to reduce their contribution to the total $3 \%$ of a radiation length value for the completed SVX detector for angles perpendicular to the beamline. In fact, each assembled SVX barrel will weigh less than $1.0 \mathrm{~kg}$. Besides being low mass, the bulkheads have been machined very accurately [11] and typically the four circles on which the ladders are mounted have been made concentric to within $15 \mu \mathrm{m}$.

The final assembly of ladders into the bulkheads is done on a rotating shaft assembly using a CMM. The strips of the silicon detectors are measured directly with a TV camera to an accuracy of $2.5 \mu \mathrm{m}$. The layer of ladders nearest and farthest from the beamline are oriented so that the detector strips face the beamline and thus measurements can be made only of the back edges and corners of the detectors after assembly into the barrel. For these two layers, the edges and corners have been referenced to the detector strip locations 


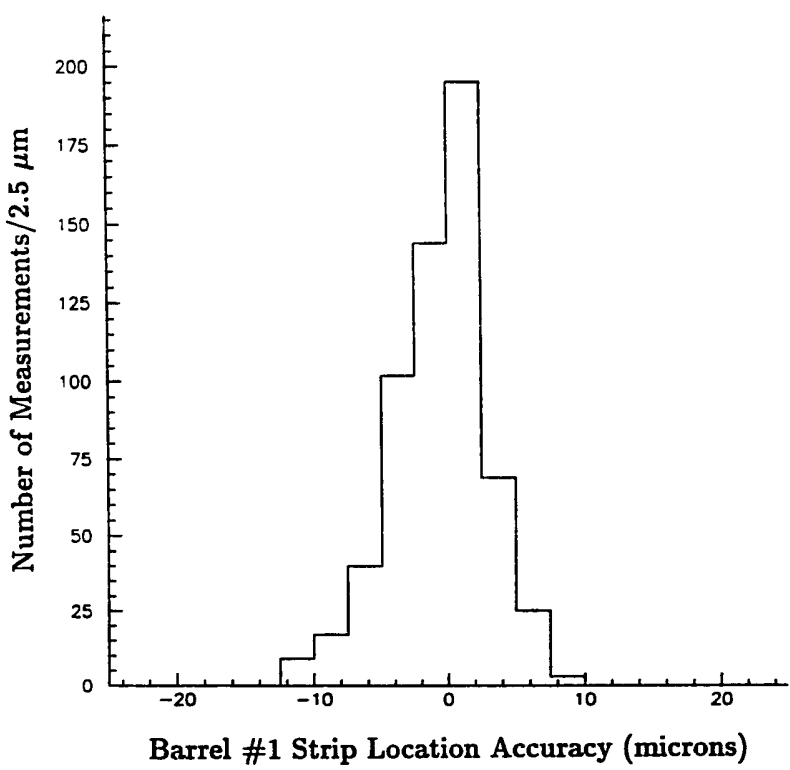

Fig. 4. Uncertainty in the position of the silicon strip detectors mounted in the first SVX barrel.

from measurements done prior to installation. Fig. 4 shows the repeatability in the measurement of the position of the silicon detector strips for all ladders mounted in the first barrel. This distribution was determined by taking the difference of two measurements of the detector strip positions during the barrel construction and has an rms of $3.6 \mu \mathrm{m}$, which is consistent with the repeatability of the measuring device. In addition to the strip measurements, the bow or radius of each ladder is measured along its length using the CMM. Fig. 5 shows the maximum bow or sagitta of all ladders in one-half of the SVX detector. The average value is

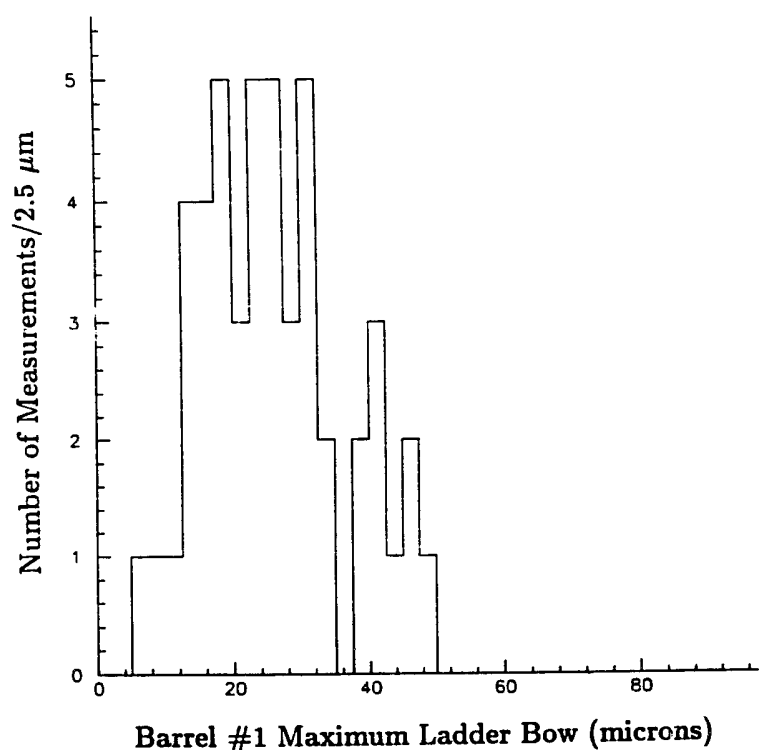

Fig. 5. Distribution of the maximum bow or sagitta for all ladders in the first SVX barrel.
$26 \mu \mathrm{m}$ and will have a negligible contribution to the SVX detector position resolution.

After the ladders are installed between the bulkheads, a lightweight composite cylinder, consisting of 1 $\mathrm{mm}$ of Rohacell wrapped in $75 \mu \mathrm{m}$ of aluminum foil, slides over the barrel and is glued to both bulkheads. This cylinder and its connection to the bulkheads serve to maintain the internal mechanical alignment of the ladders after the barrel is removed from the assembly fixture and also provides shielding from stray rf fields. In addition, it isolates the SVX detector both thermally and electrically from the surrounding tracking chambers. The SVX detector is then mounted inside in the CDF vertex time projection chamber using a 3-point kinematic support to minimize the mechanical distortion effects of any thermal gradients in the detector. The inner layer of silicon is at a radius of 1.177 in. from the beam and is thus $<0.5$ in. from the surface of the 1.5 in. $0 . d .0 .020$ in. wall thickness beryllium beam pipe.

\section{SVX cooling system}

The goal of the low mass SVX cooling system is to remove heat from the readout electronics located inside the SVX detector and to intercept heat from the surrounding tracking chamber electronics in order to keep the silicon strip detectors and the mechanical structure of the barrel at the ladder installation temperature of $20^{\circ} \mathrm{C}$. This is necessary to avoid the increase in silicon strip leakage current from higher temperature operation and to minimize thermal gradients in the internal detector structure so that the initial high-quality mechanical alignment can be maintained.

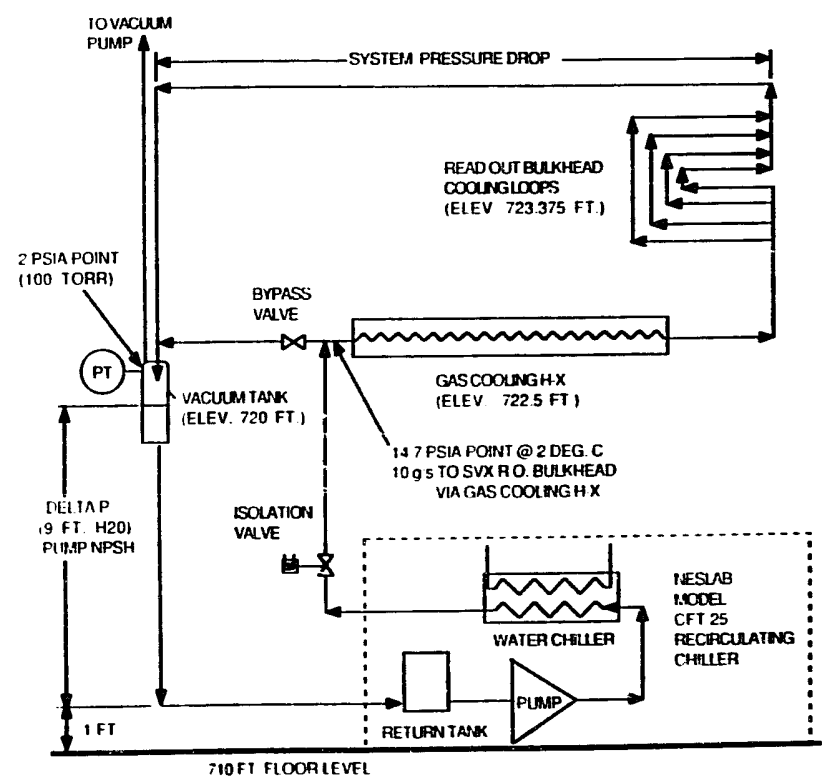

Fig. 6. SVX cooling system flow diagram. 


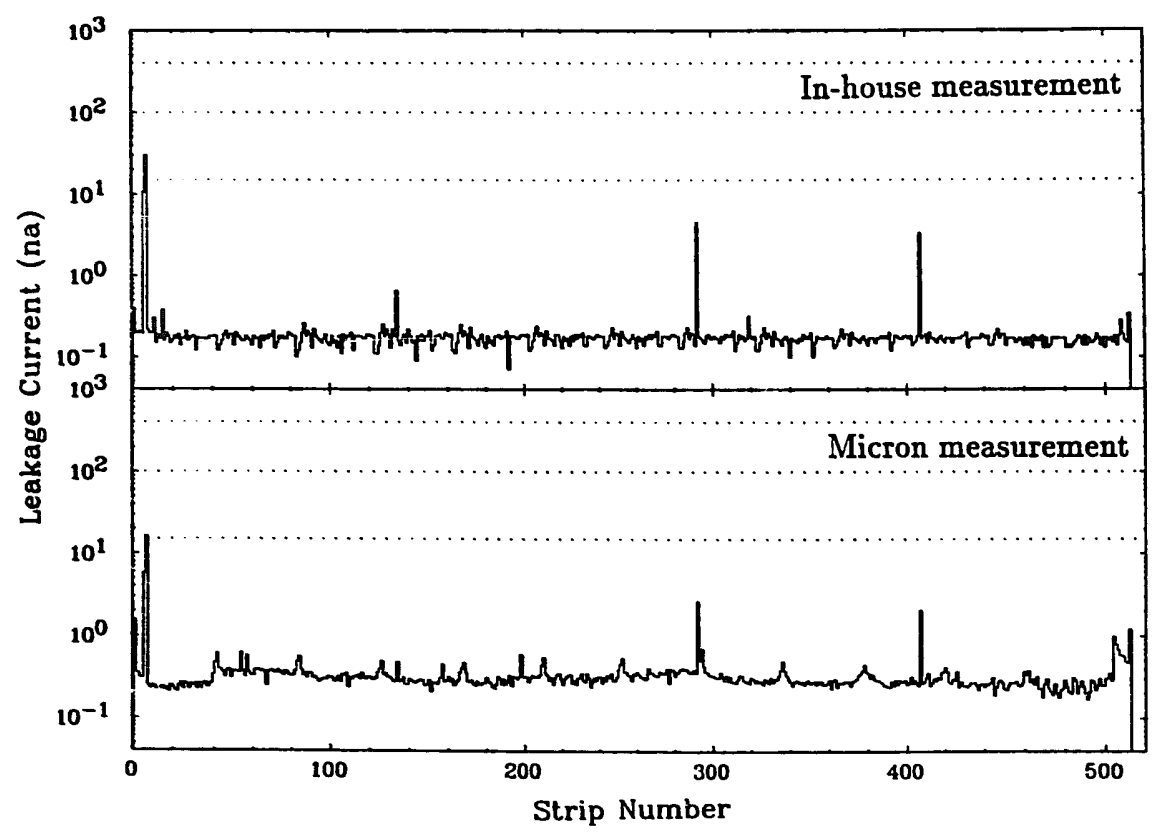

Fig. 7. Leakage current on a typical 512 strip detector used in the CDF SVX, measured both by the manufacturer and in-house.
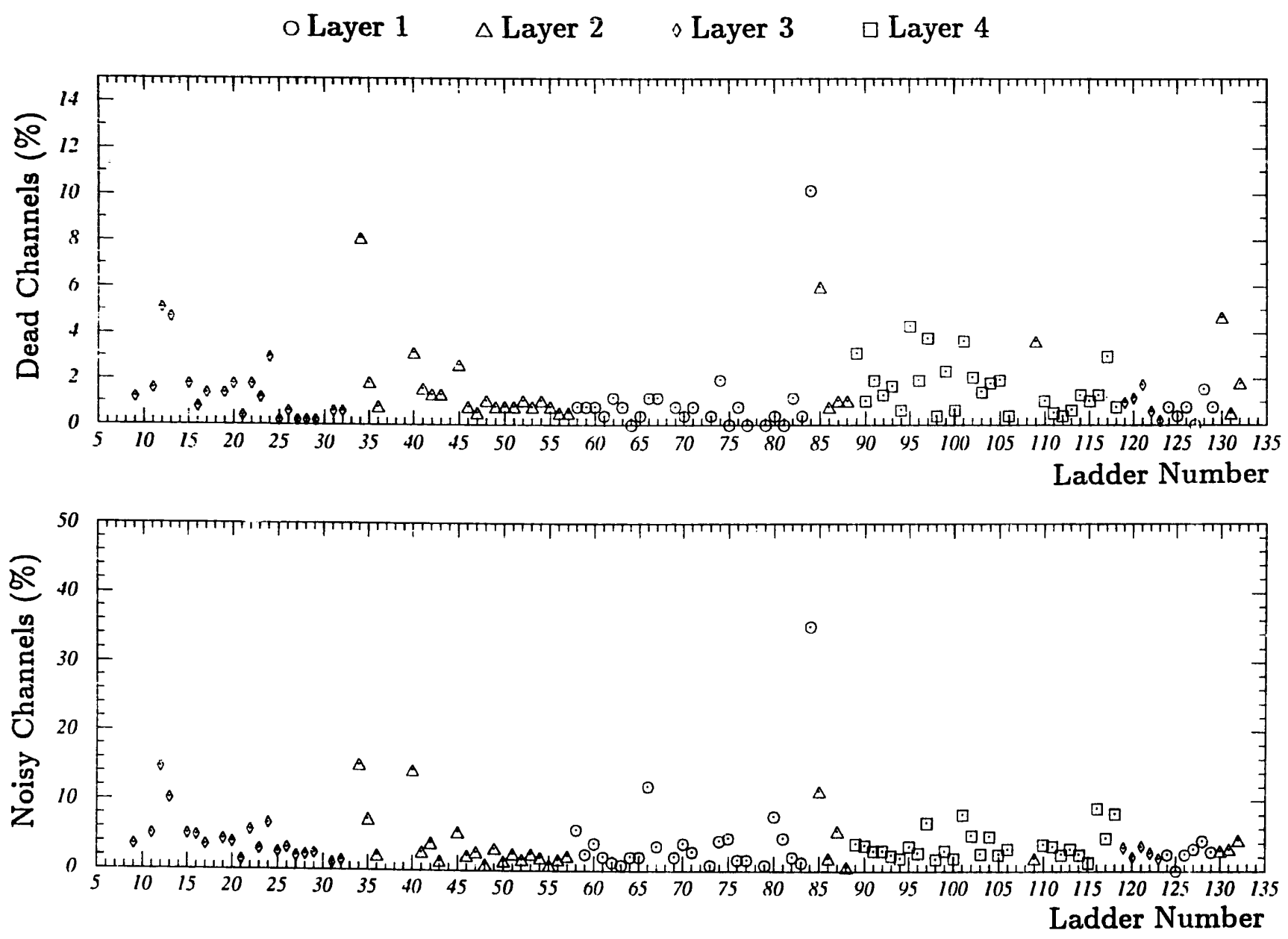

Fig. 8. Noisy and dead channels (in percent) after all tests for ladders constructed for use in the SVX. 
Fig. 6 shows in general terms the layout of the cooling system. Each layer of the bulkhead has a cooling loop consisting of $3 / 32$ in. i.d. 0.014 in. wall thickness aluminum piping containing $5^{\circ} \mathrm{C}$ water flowing at a rate of $2 \mathrm{~g} / \mathrm{s}$ to remove heat from the SVXD readout chip. The piping is in thermal contact with the beryllium bulkhead and runs underneath the ledge on which the readout circuit board is mounted. The number of readout chips varies from 2 to 6 per ladder for the 4 layers of silicon and the heat load is $175 \mathrm{~mW} / \mathrm{chip}$, giving a total heat load from the readout chips of 31.5 W per barrel. An additional cooling circuit is used to remove heat from the port card, which due to its power regulating and signal driving functions, generates 1800 $\mathrm{mW}$ each or $21.6 \mathrm{~W}$ per barrel. Thus, a total of $53.1 \mathrm{~W}$ of heat is generated by the electronics in each half of the SVX.

In addition to the water cooling, gas cooling is introduced at the opposite end of the SVX detector in order to reduce thermal gradients across the detector since most of the heating and cooling is occurring only at the readout end. In order to be compatible with the surrounding tracking chamber gas, argon-ethane gas is pre-cooled to $7^{\circ} \mathrm{C}$ with a heat exchanger before delivery through a low mass Rohacell and aluminum foil composite manifold into the SVX detector at a flow rate of 10 SCFH per barrel.

A special safety feature of the cooling system is that it operates at sub-atmospheric pressure inside the CDF detector volume. If a water leak were to occur, the result would be gas entering the system rather than water leaving it and this situation would be immediately detectable. Tests show that the combined water and gas cooling system is able to minimize the thermal gradients across the detecior.

\section{Silicon detector performance}

The silicon strip detectors [12] used in the CDF SVX detector are single-sided and dc-coupled to the SVXD readout chip. Fig. 7 shows the leakage current as a function of strip number for a typical detector with 512 strips. The baseline leakage current is usually $<0.5 \mathrm{nA}$. After being mounted onto a ladder and wirebonded to the SVXD chip, the three detectors on
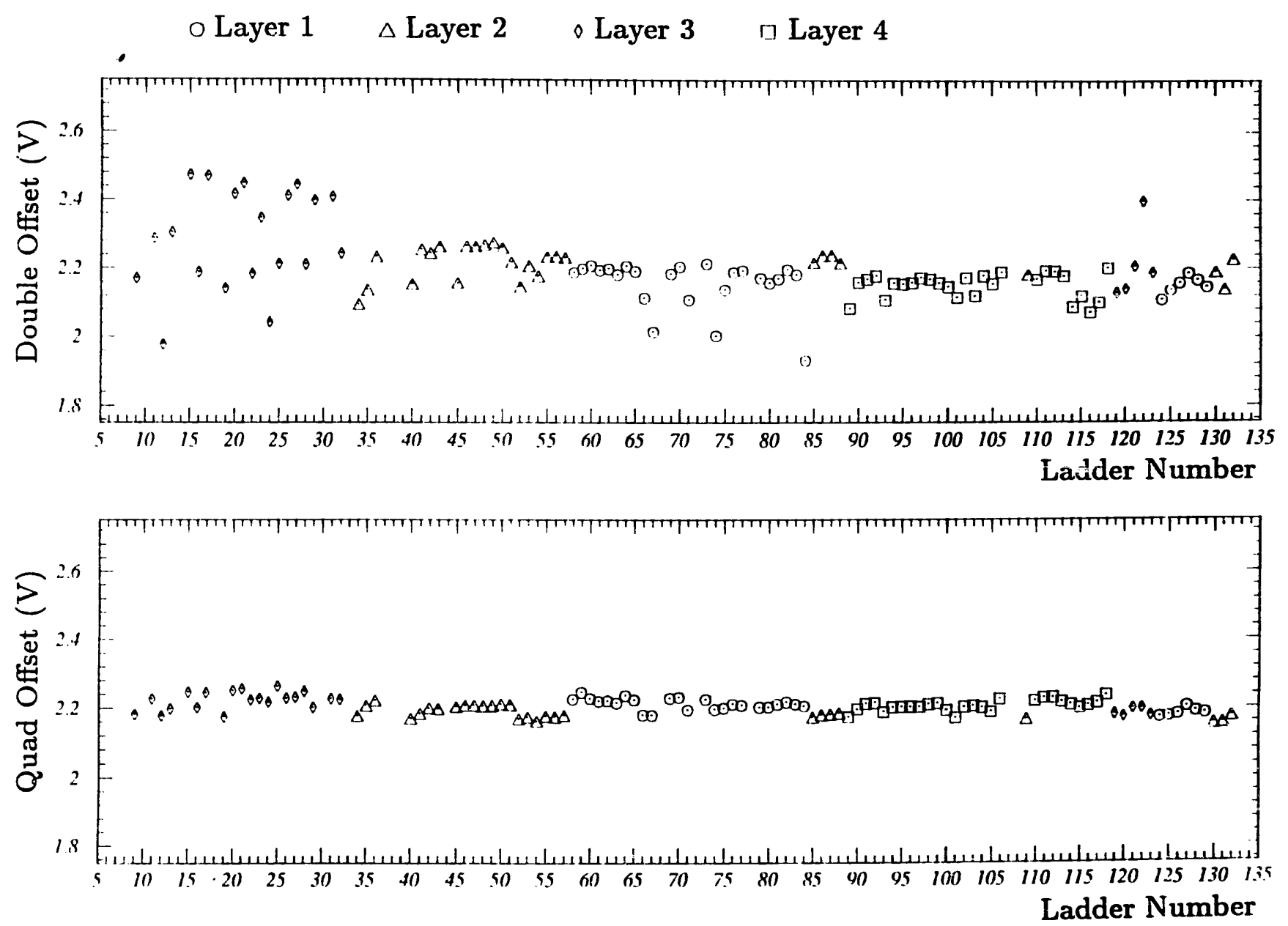

Fig. 9. Double and quadruple sample and hold offset voltages for ladders to be used in the SVX. 
a ladder undergo various calibration tests, such as charge injection calibration and sparse threshold calibration. Fig. 8 shows the percentage of noisy and dead channels, by layer number, after all tests for ladders constructed for use in the SVX. The criteria for a noisy channel is that the strip will be efficient for charged particle detection but that it will be read out more than $10 \%$ of the time for a sparse threshold value which is three standard deviations below the average value for that ladder. It can be seen that the number of noisy channels is at the $2-3 \%$ level for most ladders. The ladders with the lowest number of noisy and dead channels were then selected for installation into the barreis. The result is that the number of dead channels is measured to be $<1.5 \%$ on average and after all testing, $>98.5 \%$ of the strips are fully functioning on the SVX detector.

In addition to the above tests, the operation of the quadruple sample and hold circuitry was tested on all ladders. This circuitry, which integrates the charge signal from a strip both before and during the beam crossing, should remove the differing offset voltages due to leakage current variation for each charge integrating amplifier on the readout chip. Fig. 9 shows the expected leveling effect on the offset voltages in the quadruple sample and hold operation as compared to the double sample and hold. This is an important confirmation of a major operational function of the SVXD readout chip.

\section{Conclusions}

The work is nearing completion on the construction of the CDF silicon vertex detector. Initial results show that the internal mechanical alignment is on order 10 $\mu \mathrm{m}$ and that $>98.5 \%$ of the silicon strips are fully functional. Data-taking is scheduled to begin in early
1992 and the installation of the CDF silicon vertex detector coulu play a major role in high energy physics research for the next several years.

\section{References}

[1] S. Dell'Agnello et al., these Proceedings, Proc. 5th Pisa Meeting on Advanced Detectors: Frontier Detectors for Frontier Physics, La Biodola, Isola d'Elba, Italy, May 26-31, 1991, eds. A. Baldini, A. Scribano and G. Tonelli, Nucl. Instr. and Meth. A315 (1992) 188.

[2] N. Bacchetta et al., Radiation Damage Measurement on the SVX Readout Chip, presented at this conference (5th Pisa Meeting on Advanced Detectors: Detectors for Frontier Physics, La Biodola, Isola d'Elba, Italy, 26-31 May, 1991, eds. A. Baldini, A. Scribano and G. Tonelli).

[3] W.C. Carithers et al., Nucl. Instrum. and Methods A289 (1990) 388.

[4] Manufactured by Rohm Tech, Inc., 195 Canal Street, Malden, MA 02148 USA.

[5] Cordax 1808-MH coordinate measuring machine manufactured by Sheffield Measurement, P.O. Box 1127, Dayton, OH 45401 USA.

[6] Hexcel 5313 epoxy with hardener "B" manufactured by Hexcel Corp., 20701 Nordhoff Street, Chatsworth. CA 91311 USA.

[7] Supplied by Stellar Industries, 225 Viscoloid Avenue, Leominster, MA 01453 USA.

[8] S.A. Kleinfelder et al., IEEE Trans. Nucl. Sci. NS-35 (1988) 171.

[9] C. Haber et al., IEEE Trans. Nucl. Sci. NS-37 (1990) 1120.

[10] Manufactured by the Hughes Aircraft Co., Connecting Devices Division, 17150 Von Karman Avenue, Irvine, CA 92714 USA

[11] Fabricated by Hardric Laboratories, 1490 Main Street, Waltham, MA 02154 USA.

[12] Manufactured by Micron Semiconductor LTD., 1 Royal Buildings, Marlborough Road, Churchill Ind. Estate, Lancing, Sussex BN15 8UN, UK. 